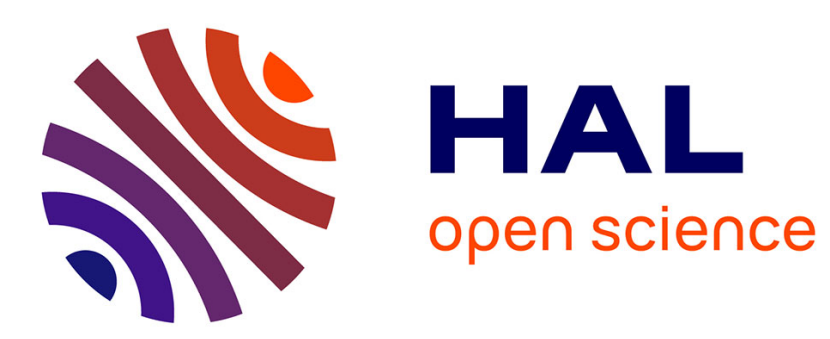

\title{
A Hygro-Elastic Self-Consistent Model for Fiber-Reinforced Composites
}

\author{
Frédéric Jacquemin, Sylvain Fréour, Ronald Guillén
}

\section{To cite this version:}

Frédéric Jacquemin, Sylvain Fréour, Ronald Guillén. A Hygro-Elastic Self-Consistent Model for FiberReinforced Composites. Journal of Reinforced Plastics and Composites, 2005, 24 (7), pp.485-502. 10.1177/0731684405045014 . hal-01004838

\section{HAL Id: hal-01004838 \\ https://hal.science/hal-01004838}

Submitted on 19 Oct 2017

HAL is a multi-disciplinary open access archive for the deposit and dissemination of scientific research documents, whether they are published or not. The documents may come from teaching and research institutions in France or abroad, or from public or private research centers.
L'archive ouverte pluridisciplinaire HAL, est destinée au dépôt et à la diffusion de documents scientifiques de niveau recherche, publiés ou non, émanant des établissements d'enseignement et de recherche français ou étrangers, des laboratoires publics ou privés. 


\title{
A Hygroelastic Self-consistent Model for Fiber-reinforced Composites
}

\author{
F. JACQUEMIN, * S. FRÉOUR AND R. GUILLÉN \\ Institut de Recherche en Génie Civil et Mécanique (UMR CNRS 6183) \\ IUT de Saint-Nazaire, Boulevard de l'Université \\ BP 406, 44602 Saint-Nazaire, France
}

\begin{abstract}
Stress analyses are performed in unidirectional fiber-reinforced composites, exposed to ambient fluid, by extending a classical self-consistent model to hygroelastic solicitations. Constitutive laws are given for the macroscopic elastic properties and Coefficients of Moisture Expansion (CME) by considering a jump in moisture content between the fiber and the matrix. Inverse forms for the unknown CME of the constituent matrix are proposed. The macroscopic (ply) and local (fiber and matrix) internal stress states are evaluated for various moisture content ratios between the matrix and the ply. The macroscopic stresses are calculated by using continuum mechanics formalisms and the local stresses are deduced from the scale transition model.
\end{abstract}

KEY WORDS: self-consistent model, moisture expansion coefficients, hygroelastic stresses.

\section{INTRODUCTION}

$\mathbf{T}$ HE INCREASING DEPLOYMENT of composite laminates in engineering applications, because of their high strength-to-weight ratio as well as corrosion and fatigue resistance, has given greater attention to the long-term behavior of composite components in hygroscopic environments. Composite materials are an increasingly common alternative to traditional materials such as metals and polymers. Now, contrary to the metallic materials or metal-matrix composites, carbon-epoxy composites can absorb significant amount of water. Moreover, the moisture contents of the epoxy resin and carbon fibers are strongly different, because carbon fibers are generally considered not to absorb water. In addition, carbon fibers and epoxy resin are characterized by heterogeneous Coefficients of Moisture Expansion (CME). In consequence, internal stresses rise from hygroscopic solicitations of composites. Now, the knowledge of internal stresses is necessary to predict a possible damage occurrence in the material during its manufacturing process or service life. Thus, the study of the development of internal stresses due to hygroelastic solicitations in composites is very important.

Numerous papers available in the literature deal with this question, using Finite Element Analysis or Continuum Mechanics-based formalisms. These methods allow the calculation of the macroscopic stresses in each ply constituting the composites. However,

*Author to whom correspondence should be addressed. E-mail: jacquemin @lamm.univ-nantes.fr 
they do not provide information on the local mechanical states, in the fibers and matrix of a given ply, and, consequently, do not explain the phenomenon of matrix cracking and damage development in composite structures. The present work is precisely focused on the study of the internal stresses in the constituents of the ply. In order to reach this goal, scale transition models are required.

In the second section, the classical elastic self-consistent scale transition model is extended in order to predict the multiscale hygroelastic behavior of composite materials. The formalism described takes into account the heterogeneities of moisture content between the matrix and fibers. Constitutive laws are given for the macroscopic elastic properties and the CME of the ply. Inverse forms are proposed too. They provide the determination of the unknown CME of a given epoxy resin.

In the third part of this article, closed-form solutions are given for the calculation of the transient moisture concentration and the induced macroscopic internal stresses in each ply constituting a composite material exposed to ambient fluid.

Finally, applications of the multiscale approach are given in the fourth section. Elasticity constants and CME predicted by the self-consistent model are compared to reference values, respectively Reuss-Voigt estimates and the Tsai-Hahn model. Mechanical states, at both macroscopic (ply) and pseudo-macroscopic (fiber or matrix) scales are evaluated for various conditions, especially in terms of moisture contents. Results obtained are compared to a macroscopic strength criterion in order to predict the damage occurrence in the simulated composite material.

\section{SELF-CONSISTENT ESTIMATES FOR HYGROELASTIC BEHAVIOR OF COMPOSITES}

\section{Extension of the Classical Self-consistent Model to Hygroelastic Solicitations}

Self-consistent models based on the mathematical formalism proposed by Kröner [1] constitute a reliable method to predict the micromechanical behavior of heterogeneous materials. The method was initially introduced to treat the case of polycrystalline materials, i.e. duplex steels, aluminum alloys, etc., submitted to purely elastic solicitations. Estimations of homogenized elastic properties and related problems are given in several works [2-4]. The model was extended by Hutchinson [5] to thermoelastic solicitations and gave satisfactory results on either single-phase [6,7] or two-phase [8] materials. In the present paper, the classical model is improved in order to take into account stresses and strains due to moisture in carbon fiber-reinforced polymer-matrix composites.

In the model, ellipsoidal inclusions are embedded in an infinite medium, called Homogeneous Effective Medium or HEM. HEM is assumed to behave as one particular ply: its hygroelastic properties come from homogenization operations performed on the hygroelastic constants of the matrix and fibers constituting the considered ply. The material is investigated at two different scales for the needs of micromechanical modeling:

- the average behavior of a ply, represented by the HEM, defines the macroscopic scale of the model. It is denoted by the superscript $I$.

- the properties and mechanical states of the matrix and fiber are respectively indicated by the superscripts $m$ and $f$. These constituents define the so-called "pseudomacroscopic" scale of the material [9]. 
Within this approach, the hygrothermal dilatation generated by a moisture content increment $\Delta C^{I}$ is treated as a transformation strain. The macroscopic stresses $\sigma$ are given by:

$$
\boldsymbol{\sigma}^{I}=\mathbf{L}^{I}:\left(\varepsilon^{I}-\boldsymbol{\beta}^{I} \Delta C^{I}\right)
$$

where $\mathbf{L}$ is the elasticity tensor, $\boldsymbol{\varepsilon}$ the strains due to elastic and hygrothermal solicitations, and $\beta$ the coefficients of moisture expansion.

At pseudo-macroscopic scale, similar equations can be written:

$$
\boldsymbol{\sigma}^{\alpha}=\mathbf{L}^{\alpha}:\left(\boldsymbol{\varepsilon}^{\alpha}-\boldsymbol{\beta}^{\alpha} \Delta C^{\alpha}\right)
$$

where $\alpha$ replaces the appropriate superscript (i.e. $f$ or $m$ ) for the carbon fiber or polymer matrix. One should notice that contrary to the thermoelastic self-consistent model, where the temperature increment $\Delta T$ is generally considered homogeneous at every scale, the moisture content increment is often substantially different in the fiber and the matrix.

In a fundamental work [10], Eshelby demonstrated that when the elementary inclusions (here the matrix and the fiber) were assumed to have ellipsoidal shapes, stresses and strains were related by the following scale transition relation:

$$
\boldsymbol{\sigma}^{\alpha}-\boldsymbol{\sigma}^{I}=-\mathbf{L}^{I}: \mathbf{R}^{I}:\left(\boldsymbol{\varepsilon}^{\alpha}-\boldsymbol{\varepsilon}^{I}\right)
$$

$\mathbf{R}^{I}$ is the reaction tensor. It satisfies:

$$
\mathbf{R}^{I}=\left(\mathbf{I}-\mathbf{S}_{\mathrm{esh}}^{I}\right): \mathbf{S}_{\mathrm{esh}}^{I^{-1}}=\left(\mathbf{L}^{I^{-1}}-\mathbf{E}^{I}\right): \mathbf{E}^{I^{-1}}
$$

In Equation (4), I stands for the fourth-order identity tensor. Hill's tensor $\mathbf{E}^{I}$ expresses the dependence of the reaction tensor on the morphology assumed for the matrix and its reinforcements [11]. It can be expressed as a function of Eshelby tensor, through $\mathbf{E}^{I}=\mathbf{L}^{I}: \mathbf{S}_{\mathrm{esh}}^{I}$.

In another paper, Hill demonstrated the following useful average equations [12]:

$$
\begin{aligned}
\left\langle\boldsymbol{\sigma}^{\alpha}\right\rangle_{\alpha=f, m} & =\boldsymbol{\sigma}^{I} \\
\left\langle\boldsymbol{\varepsilon}^{\alpha}\right\rangle_{\alpha=f, m} & =\boldsymbol{\varepsilon}^{I}
\end{aligned}
$$

\section{Prediction of the Homogenized Hygroelastic Properties of a Ply}

In the classical Self-consistent (SC) framework, the properties of the elementary constituents are supposed to be known. On the contrary, the average properties of the material at the macroscopic scale are generally deduced from calculation. The present section aims to find expressions for the homogenized CME $\boldsymbol{\beta}^{I}$ of a ply.

Using (1)-(4), we easily obtain a linear relation between pseudo-macroscopic and macroscopic strains:

$$
\boldsymbol{\varepsilon}^{\alpha}=\left(\mathbf{L}^{\alpha}+\mathbf{L}^{I}: \mathbf{R}^{I}\right)^{-1}:\left[\left(\mathbf{L}^{I}+\mathbf{L}^{I}: \mathbf{R}^{I}\right): \boldsymbol{\varepsilon}^{I}+\mathbf{L}^{\alpha}: \boldsymbol{\beta}^{\alpha} \Delta C^{\alpha}-\mathbf{L}^{I}: \boldsymbol{\beta}^{I} \Delta C^{I}\right]
$$


The introduction of Hill's average relation (5) in (6) yields:

$$
\boldsymbol{\varepsilon}^{I}=\left\langle\left(\mathbf{L}^{\alpha}+\mathbf{L}^{I}: \mathbf{R}^{I}\right)^{-1}:\left[\left(\mathbf{L}^{I}+\mathbf{L}^{I}: \mathbf{R}^{I}\right): \boldsymbol{\varepsilon}^{I}+\mathbf{L}^{\alpha}: \boldsymbol{\beta}^{\alpha} \Delta C^{\alpha}-\mathbf{L}^{I}: \boldsymbol{\beta}^{I} \Delta C^{I}\right]\right\rangle_{\alpha=f, m}
$$

The previous equation develops as follows:

$$
\begin{aligned}
\boldsymbol{\varepsilon}^{I}= & \left\langle\left(\mathbf{L}^{\alpha}+\mathbf{L}^{I}: \mathbf{R}^{I}\right)^{-1}:\left(\mathbf{L}^{I}+\mathbf{L}^{I}: \mathbf{R}^{I}\right)\right\rangle_{\alpha=f, m}: \boldsymbol{\varepsilon}^{I} \\
& +\left\langle\left(\mathbf{L}^{\alpha}+\mathbf{L}^{I}: \mathbf{R}^{I}\right)^{-1}:\left[\mathbf{L}^{\alpha}: \boldsymbol{\beta}^{\alpha} \Delta C^{\alpha}-\mathbf{L}^{I}: \boldsymbol{\beta}^{I} \Delta C^{I}\right]\right\rangle_{\alpha=f, m}
\end{aligned}
$$

Since this relation must be satisfied for any hygromechanical state $\left\{\boldsymbol{\varepsilon}^{I}, \Delta C^{I}, \Delta \mathrm{C}^{f}, \Delta C^{m}\right\}$, the first term of the right member of (8) must be equal to $\mathbf{I}$, while the second term must be null:

$$
\begin{gathered}
\left\langle\left(\mathbf{L}^{\alpha}+\mathbf{L}^{I}: \mathbf{R}^{I}\right)^{-1}:\left(\mathbf{L}^{I}+\mathbf{L}^{I}: \mathbf{R}^{I}\right)\right\rangle_{\alpha=f, m}=\mathbf{I} \\
\left\langle\left(\mathbf{L}^{\alpha}+\mathbf{L}^{I}: \mathbf{R}^{I}\right)^{-1}:\left[\mathbf{L}^{\alpha}: \boldsymbol{\beta}^{\alpha} \Delta C^{\alpha}-\mathbf{L}^{I}: \boldsymbol{\beta}^{I} \Delta C^{I}\right]\right\rangle_{\alpha=f, m}=0
\end{gathered}
$$

Equation (9) yields the very classical self-consistent estimate for the macroscopic elastic stiffness (e.g. an equivalent form is given in [4]):

$$
\mathbf{L}^{I}=\left\langle\left(\mathbf{L}^{\alpha}+\mathbf{L}^{I}: \mathbf{R}^{I}\right)^{-1}:\left(\mathbf{L}^{I}+\mathbf{L}^{I}: \mathbf{R}^{I}\right): \mathbf{L}^{\alpha}\right\rangle_{\alpha=f, m}
$$

Moreover, starting from (10), elementary algebraic manipulations lead to the homogenized CME for the composite:

$$
\left\langle\left(\mathbf{L}^{\alpha}+\mathbf{L}^{I}: \mathbf{R}^{I}\right)^{-1}: \mathbf{L}^{\alpha}: \boldsymbol{\beta}^{\alpha} \Delta C^{\alpha}\right\rangle_{\alpha=f, m}=\left\langle\left(\mathbf{L}^{\alpha}+\mathbf{L}^{I}: \mathbf{R}^{I}\right)^{-1}: \mathbf{L}^{I}: \boldsymbol{\beta}^{I} \Delta C^{I}\right\rangle_{\alpha=f, m}
$$

$\mathbf{L}^{I}, \boldsymbol{\beta}^{I}$, and $\Delta C^{I}$ being constants over the pseudo-macroscopic elements, we may extract them from the summation:

$$
\left\langle\left(\mathbf{L}^{\alpha}+\mathbf{L}^{I}: \mathbf{R}^{I}\right)^{-1}\right\rangle_{\alpha=f, m}^{-1}:\left\langle\left(\mathbf{L}^{\alpha}+\mathbf{L}^{I}: \mathbf{R}^{I}\right)^{-1}: \mathbf{L}^{\alpha}: \boldsymbol{\beta}^{\alpha} \Delta C^{\alpha}\right\rangle_{\alpha=f, m}=\mathbf{L}^{I}: \boldsymbol{\beta}^{I} \Delta C^{I}
$$

Finally, we obtain an explicit form for the macroscopic CME:

$$
\boldsymbol{\beta}^{I}=\frac{1}{\Delta C^{I}} \mathbf{L}^{I^{-1}}\left\langle\left(\mathbf{L}^{\alpha}+\mathbf{L}^{I}: \mathbf{R}^{I}\right)^{-1}\right\rangle_{\alpha=f, m}^{-1}:\left\langle\left(\mathbf{L}^{\alpha}+\mathbf{L}^{I}: \mathbf{R}^{I}\right)^{-1}: \mathbf{L}^{\alpha}: \boldsymbol{\beta}^{\alpha} \Delta C^{\alpha}\right\rangle_{\alpha=f, m}
$$


Since the increment of moisture concentration in fiber is usually null, the introduction of this additional assumption (i.e. $\Delta \mathrm{C}^{f}=0$ ) involves the following simplifications:

$$
\boldsymbol{\beta}^{I}=v^{m} \frac{\Delta C^{m}}{\Delta C^{I}} \mathbf{L}^{I^{-1}}\left\langle\left(\mathbf{L}^{\alpha}+\mathbf{L}^{I}: \mathbf{R}^{I}\right)^{-1}\right\rangle_{\alpha=f, m}^{-1}:\left(\mathbf{L}^{m}+\mathbf{L}^{I}: \mathbf{R}^{I}\right)^{-1}: \mathbf{L}^{m}: \boldsymbol{\beta}^{m}
$$

where $v^{m}$ stands for the volume fraction of matrix in the considered ply $\left(v^{m}+v^{f}=1\right)$.

When the equilibrium state is reached, the maximum moisture content of the neat resin may be estimated from the maximum moisture content of the composite. By assuming that the fibers do not absorb any moisture, $\Delta C^{I}$ and $\Delta C^{m}$ are related by the expression given by Loos and Springer [13]:

$$
\Delta C^{I}=\Delta C^{m} W^{m}
$$

where $W^{m}$ is the weight fraction (percent) of the resin in the composite.

The previous equation develops as follows:

$$
\frac{\Delta C^{m}}{\Delta C^{I}}=\frac{\rho^{I}}{v^{m} \rho^{m}}
$$

where $\rho^{I}$ and $\rho^{m}$ are respectively the composite and resin densities.

Introducing (17) in (15), the macroscopic CME are expressed:

$$
\boldsymbol{\beta}^{I}=\frac{\rho^{I}}{\rho^{m}} \mathbf{L}^{I^{-1}}\left\langle\left(\mathbf{L}^{\alpha}+\mathbf{L}^{I}: \mathbf{R}^{I}\right)^{-1}\right\rangle_{\alpha=f, m}^{-1}:\left(\mathbf{L}^{m}+\mathbf{L}^{I}: \mathbf{R}^{I}\right)^{-1}: \mathbf{L}^{m}: \boldsymbol{\beta}^{m}
$$

Actually, the theoretical relation (16) of Loos and Springer can be affected by a factor $\gamma$ $(\gamma \geq 1) . \gamma$ stands for the ratio between mass water in the composite and the matrix. The imperfect adhesion in fiber-reinforced composites plays an important role in the diffusion process. Between the constituents in a composite, one or more interphases can develop that separate the reinforcing inclusion phase from the host matrix phase [14]. Fiber debonding from the matrix induces localized water entrapment [15], hence apparently higher water uptakes. Moreover, the matrix can be porous. Taking into account this particularity leads to the following modifications for relations (16) and (17):

$$
\begin{aligned}
& \Delta C^{I}=\gamma \Delta C^{m} W^{m} \\
& \frac{\Delta C^{m}}{\Delta C^{I}}=\frac{\rho^{I}}{\gamma\left(v^{m}\right) \rho^{m}}
\end{aligned}
$$

Introducing (20) in (15), the macroscopic CME are then expressed:

$$
\boldsymbol{\beta}^{I}=\frac{\rho^{I}}{\gamma \rho^{m}} \mathbf{L}^{I^{-1}}\left\langle\left(\mathbf{L}^{\alpha}+\mathbf{L}^{I}: \mathbf{R}^{I}\right)^{-1}\right\rangle_{\alpha=f, m}^{-1}:\left(\mathbf{L}^{m}+\mathbf{L}^{I}: \mathbf{R}^{I}\right)^{-1}: \mathbf{L}^{m}: \boldsymbol{\beta}^{m}
$$




\section{Inverse Forms for the Unknown CME of the Constituent Matrix in Self-consistent Scheme}

Values for the CME of the elementary constituents of a composite (actually the one of the polymer matrix) are not always available. Using (14) or (15), one might express the CME of a given constituent of a ply. The method is based on an inversion of the scale transition homogenization relations obtained using the classical Self-consistent framework. The inversion of the generalized form obtained for (14) allows to express the unknown CME of a given constituent denoted by $m$ :

$$
\boldsymbol{\beta}^{m}=\mathbf{L}^{m^{-1}}:\left(\mathbf{L}^{m}+\mathbf{L}^{I}: \mathbf{R}^{I}\right):\left[\mathbf{A}^{I}+\mathbf{A}^{\alpha}\right]
$$

with

$$
\begin{aligned}
& \mathbf{A}^{I}=\frac{\Delta C^{I}}{\Delta C^{m}} \sum_{\alpha} \frac{v^{\alpha}}{v^{m}}\left(\mathbf{L}^{\alpha}+\mathbf{L}^{I}: \mathbf{R}^{I}\right)^{-1}: \mathbf{L}^{I}: \boldsymbol{\beta}^{I} \\
& \mathbf{A}^{\alpha}=\sum_{\alpha \neq m} \frac{v^{\alpha} \Delta C^{\alpha}}{v^{m} \Delta C^{m}}\left(\mathbf{L}^{\alpha}+\mathbf{L}^{I}: \mathbf{R}^{I}\right)^{-1}: \mathbf{L}^{\alpha}: \boldsymbol{\beta}^{\alpha}
\end{aligned}
$$

In the case of a $(f+m)$ composite, and assuming that the additional condition $\Delta C^{f}=0$ is satisfied, the CME of the matrix adopt the following form:

$$
\boldsymbol{\beta}^{m}=\frac{\Delta C^{I}}{\Delta C^{m}} \mathbf{L}^{m^{-1}}:\left(\mathbf{L}^{m}+\mathbf{L}^{I}: \mathbf{R}^{I}\right): \sum_{\alpha=f, m} \frac{v^{\alpha}}{v^{m}}\left(\mathbf{L}^{\alpha}+\mathbf{L}^{I}: \mathbf{R}^{I}\right)^{-1}: \mathbf{L}^{I}: \boldsymbol{\beta}^{I}
$$

\section{Reuss-Voigt Model for the Estimation of the Macroscopic Elastic Moduli and Tsai-Hahn Model for the Estimation of the Macroscopic CME}

REUSS-VOIGT MODEL

Since they delimit the interval where the homogenized macroscopic elastic properties must be, the upper and lower bounds given by Reuss and Voigt assumptions are of main interest. The Voigt's assumption of uniform strains $\left(\varepsilon^{I}=\varepsilon^{\alpha}\right)$ [16], leads to the upper bound $\mathbf{L}_{V}^{I}$ and the Reuss' assumption of uniform stresses $\left(\boldsymbol{\sigma}^{I}=\boldsymbol{\sigma}^{\alpha}\right)$ [17], leads to the lower bound $\mathbf{L}_{R}^{I}$ :

$$
\left.\left.\mathbf{L}_{V}^{I}=\left\langle\mathbf{L}^{\alpha}\right\rangle_{\alpha=f, m}\right\rangle \mathbf{L}^{I}\right\rangle \mathbf{L}_{\mathbf{R}}^{I}=\left\langle\mathbf{L}^{\alpha^{-1}}\right\rangle_{\alpha=f, m}^{-1}
$$

TSAI-HAHN MODEL

Reuss and Voigt estimates are not appropriate to obtain values of the macroscopic CME reliable for comparisons with experiments. Actually, these models do not represent bounds for either thermal or moisture expansion in heterogeneous media such as 
composites or multiphase materials. Usually, identical models are used for the coefficients of thermal expansion and coefficients of moisture expansion $[18,19]$. Thus, these models do not take into account a jump in moisture content between the constituents. On the contrary, the Tsai-Hahn model [20], which introduces different moisture contents in the fiber and the matrix, constitutes an interesting assessment of the validity of SC estimates for CME. According to the authors, the components of the CME of the composite satisfy:

$$
\begin{gathered}
\beta_{11}^{I}=\frac{v^{f} E^{f} C^{f m} \beta^{f}+v^{m} E^{m} \beta^{m}}{\left(v^{f} E^{f}+v^{m} E^{m}\right)\left(v^{f} C^{f m} \rho^{f}+v^{m} \rho^{m}\right)} \rho^{I} \\
\beta_{22}^{I}=\frac{v^{f}\left(1+v^{f}\right) C^{f m} \beta^{f}+v^{m}\left(1+v^{m}\right) E^{m} \beta^{m}}{v^{f} C^{f m} \rho^{f}+v^{m} \rho^{m}} \rho^{I}-\left(v^{f} v^{f}+v^{m} v^{m}\right) \beta_{11}^{I}
\end{gathered}
$$

where $E$ and $v$ stand for the elastic modulus and Poisson's ratio, $\rho$ is the density, and $C^{f m}$ the ratio between moisture contents in the fiber and the matrix: $C^{f m}=C^{f} / C^{m}$.

Actually, (27) and (28) shorten since major simplifications are taken into account. Especially, in the case of carbon-epoxy composites, $C^{f}$ tends toward 0 and $E^{m} / E^{f}<<1$, we obtain:

$$
\beta_{11}^{I}=0 \quad \text { and } \quad \beta_{22}^{I}=\frac{\left(1+v^{m}\right) \beta^{m}}{\rho^{m}} \rho^{I}
$$

According to (29), the transverse CME component of the composite is higher than the one of the matrix. Actually, this result can be attributed to a higher moisture content in the epoxy matrix than in the composite material. The macroscopic strains being the average of the strains over the volume of the components, and the absence of moisture in fibers, explain that the CME of the resin should be weaker than the overall transverse CME. It will be verified in the following, that the Self-consistent model is able to give similar results.

Introducing the ratio between mass water in the composite and the matrix and assuming that the fibers do not absorb any moisture, the Tsai-Hahn estimation of macroscopic CME is:

$$
\begin{gathered}
\beta_{11}^{I}=\frac{E^{m} \beta^{m}}{\left(v^{f} E^{f}+v^{m} E^{m}\right)} \frac{\rho^{I}}{\gamma \rho^{m}} \\
\beta_{22}^{I}=\left(1+v^{m}\right) \beta^{m} \frac{\rho^{I}}{\gamma \rho^{m}}-\left(v^{f} v^{f}+v^{m} v^{m}\right) \beta_{11}^{I}
\end{gathered}
$$

\section{MACROSCOPIC ANALYSIS}

\section{Moisture Concentration}

Consider an initially dry, thin unidirectionally reinforced composite pipe, whose inner and outer radii are $a$ and $b$, respectively, and let the laminate be exposed to an ambient fluid with boundary concentration $c_{0}$. The macroscopic moisture concentration, $c^{I}(r, t)$, 
is a solution of the following system with Fick's equation (32), where $D^{I}$ is the transverse diffusion coefficient of the composite. Boundary and initial conditions are described in (33):

$$
\begin{gathered}
\frac{\partial c^{I}}{\partial t}=D^{I}\left[\frac{\partial^{2} c^{I}}{\partial r^{2}}+\frac{1}{r} \frac{\partial c^{I}}{\partial r}\right], \quad a<r<b \\
\left\{\begin{array}{l}
c^{I}(a, t)=c_{0} \text { and } c^{I}(b, t)=c_{0} \\
c^{I}(r, 0)=0
\end{array}\right.
\end{gathered}
$$

Applying the Laplace transform to the latter system and using the residue theory to express the solution in time space [21], we finally obtain the macroscopic moisture concentration:

$$
c^{I}(\bar{r}, \tau)=c_{0}+\sum_{m=1}^{\infty} \frac{2 \exp \left(-\omega_{m}^{2} \tau\right)}{\omega_{m} \Delta_{u}^{\prime}\left(\omega_{m}\right)}\left\{A_{m} J_{0}\left(\omega_{m} \bar{r}\right)+B_{m} Y_{0}\left(\omega_{m} \bar{r}\right)\right\}
$$

where $J_{0}$ and $Y_{0}$ are the Bessel's functions of order zero, $\Delta_{u}$ is the determinant of $2 \times 2$ matrix $[a] . A_{m}$ and $B_{m}$ are the determinants of matrices deduced from [a] by substituting respectively columns 1 and 2 by the constant vector $\{g\} . \Delta_{u}^{\prime}\left(\omega_{m}\right)$ is the derivative of $\Delta_{u}$ with respect to $\omega$ calculated for $\omega_{m}$ the $m$ th positive root of $\Delta_{u} . \bar{r}$ and $\tau$ are defined by the relations $\bar{r}=r / b$ and $\tau=\left(D^{I} t\right) / b^{2}$.

Furthermore, the elements of $[a]$ and $\{g\}$ are:

$$
\begin{aligned}
& a_{11}=J_{0}(\omega \bar{a}), \quad a_{12}=Y_{0}(\omega \bar{a}), \quad a_{21}=J_{0}(\omega), \quad a_{22}=Y_{0}(\omega), \\
& g_{1}=c_{0}, \quad g_{2}=c_{0} .
\end{aligned}
$$

\section{Macroscopic Stresses}

At the initial time, let us assume that the pipe is stress free. Therefore, the hygroelastic orthotropic behavior can be written as in (35)-(36), where $\boldsymbol{\beta}^{I}$ and $\mathbf{L}^{I}$ are respectively the in-plane tensors of hygroscopic expansion coefficients and moduli. Those tensors are assumed to be material constants.

$$
\begin{aligned}
&\left\{\begin{array}{c}
\boldsymbol{\sigma}_{11}^{I} \\
\boldsymbol{\sigma}_{22}^{I} \\
\boldsymbol{\sigma}_{33}^{I} \\
\tau_{12}^{I}
\end{array}\right\}=\left[\begin{array}{llll}
L_{11}^{I} & L_{12}^{I} & \mathrm{~L}_{12}^{I} & 0 \\
L_{12}^{I} & L_{22}^{I} & L_{23}^{I} & 0 \\
L_{12}^{I} & L_{23}^{I} & L_{22}^{I} & 0 \\
0 & 0 & 0 & L_{66}^{I}
\end{array}\right] \quad\left\{\begin{array}{c}
\varepsilon_{11}^{I}-\beta_{11}^{I} \Delta C^{I} \\
\varepsilon_{22}^{I}-\beta_{22}^{I} \Delta C^{I} \\
\varepsilon_{33}^{I}-\beta_{22}^{I} \Delta C^{I} \\
\gamma_{12}^{I}-\beta_{12}^{I} \Delta C^{I}
\end{array}\right\} \\
&\left\{\begin{array}{c}
\tau_{32}^{I} \\
\tau_{13}^{I}
\end{array}\right\}=\left[\begin{array}{cc}
L_{44}^{I} & 0 \\
0 & L_{55}^{I}
\end{array}\right] \quad\left\{\begin{array}{c}
\gamma_{32}^{I} \\
\gamma_{13}^{I}
\end{array}\right\}
\end{aligned}
$$


with, $\Delta C^{I}=c^{I} / \rho^{I} . c^{I}$ and $\rho^{I}$ are respectively the macroscopic moisture concentration and the mass density of the dry material.

To solve the hygromechanical problem, it is necessary to express the strains versus the displacements along with the compatibility and equilibrium equations.

Introducing a characteristic modulus $L_{0}$, we introduce the following dimensionless variables:

$$
\overline{\boldsymbol{\sigma}}^{\mathrm{I}}=\boldsymbol{\sigma}^{\mathrm{I}} / L_{0}, \overline{\mathbf{L}}^{I}=\mathbf{L}^{I} / L_{0},\left(\bar{w}^{I}, \bar{u}^{I}, \bar{v}^{I}\right)=\left(w^{I}, u^{I}, v^{I}\right) / b
$$

Displacements with respect to 1 and 2 , respectively $\bar{u}^{I}(\bar{x}, \bar{r})$ and $\bar{v}^{I}(\bar{x}, \bar{r})$ are then deduced:

$$
\left\{\begin{array}{l}
\bar{u}^{I}(\bar{x}, \bar{r})=R_{1} \bar{x} \\
\bar{v}^{I}(\bar{x}, \bar{r})=R_{2} \bar{x} \bar{r} \\
R_{1}, R_{2} \text { are constants. }
\end{array}\right.
$$

It is worth noticing that the displacements $\bar{u}^{I}(\bar{x}, \bar{r})$ and $\bar{v}^{I}(\bar{x}, \bar{r})$ do not depend on the moisture concentration field. Finally, to obtain the through-thickness or radial component of the displacement $\bar{w}^{I}$, we shall consider in the following the analytical transient concentration (34).

The radial component of the displacement field $\bar{w}^{I}$ satisfies the following equation:

$$
\bar{r}^{2} \frac{\partial^{2} \bar{w}^{I}}{\partial \bar{r}^{2}}+\bar{r} \frac{\partial \bar{w}^{I}}{\partial \bar{r}}-\bar{w}^{I}=\frac{K_{1} \bar{r}^{2}\left(\partial \Delta C^{I}\right) /(\partial \bar{r})}{\bar{L}_{22}^{I}}
$$

with, $K_{1}=\bar{L}_{12}^{I} \beta_{11}^{I}+\bar{L}_{23}^{I} \beta_{22}^{I}+\bar{L}_{22}^{I} \beta_{22}^{I}$.

It is shown that the general solution of Equation (38) can be written as the sum of a solution of the homogeneous equation and of a particular solution [22].

$$
\begin{aligned}
\bar{w}^{I}(\bar{r})= & R_{3} \bar{r}+\frac{R_{4}}{\bar{r}} \\
& -\sum_{m=1}^{\infty} \frac{2 \exp \left(-\omega_{m}^{2} \tau\right)}{\omega_{m} \Delta_{u}^{\prime}\left(\omega_{m}\right)} \frac{K_{1}}{\bar{L}_{22}^{I}}\left[A_{i} \sum_{k=0}^{\infty} \frac{(-1)^{k}(1 / 2)^{2 k+1}\left(\omega_{m}\right)^{2 k+2}}{k !(k+1) !} \frac{\bar{r}^{2 k+3}}{\left((2 k+3)^{2}-1\right)}\right. \\
& +\frac{B_{i}}{\pi}\left\{\sum_{k=0}^{\infty} \frac{(-1)^{k}(1 / 2)^{2 k+1}\left(\omega_{m}\right)^{2 k+2}}{k !(k+1) !}\left[2 \ln \left(\frac{1}{2} \omega_{m}\right)-(k+1)-\psi(k+2)\right] \frac{\bar{r}^{(2 k+3)}}{\left((2 k+3)^{2}-1\right)}\right. \\
& \left.\left.+2 \sum_{k=0}^{\infty} \frac{(-1)^{k}(1 / 2)^{2 k+1}\left(\omega_{m}\right)^{2 k+2}}{k !(k+1) !}\left[\frac{\ln (\bar{r}) \bar{r}^{2 k+3}}{\left((2 k+3)^{2}-1\right)}-\frac{2(2 k+3) \bar{r}^{2 k+3}}{\left((2 k+3)^{2}-1\right)^{2}}\right]-\bar{r} \ln (\bar{r})\right\}\right]
\end{aligned}
$$

Finally, the displacement field depends on four constants to be determined: $R_{i}$ for $i=1, \ldots, 4$. These four constants result from the following conditions:

- global force balance of the cylinder;

- nullity of the normal stress on the two lateral surfaces. 


\section{EXAMPLES}

\section{Application of Micromechanical Modeling for the Estimation of the Effective Properties}

\section{OVERALL ELASTIC ESTIMATES IN COMPOSITE MATERIALS}

The equivalent elastic behavior of two composite materials has been simulated using the self-consistent form (11). Several calculations have been performed for different morphologies for fiber and matrix. Transverse $\left(a_{2}\right)$ and normal $\left(a_{3}\right)$ semi-axis of the ellipsoid were set to 1 . Then, the list of the length of the longitudinal $\left(a_{1}\right)$ semi-axis considered for the calculations is the following: $\{1,5,10,15,20,25,50,75,100\}$. Actually, according to [11], morphology affects the overall elastic stiffness of the material through Hill's tensor.

The aim of this numerical study is the determination of an optimum morphology in order to obtain a satisfactory equivalent macroscopic stiffness standing comparisons with the behavior commonly observed in practice for a ply. Results obtained with Self-consistent (SC) estimates for longitudinal and transverse Young's moduli, have been compared with Voigt and Reuss assumptions (see Figure 1), in the case of two materials: a carbon-epoxy composite, T300/5208, and a Metal-Matrix Composite (MMC) Al-SiC. The behavior of each composite is governed by its constituents, i.e. the properties of the fibers, the surrounding matrix, and the relative amount of the fibers and matrix in the
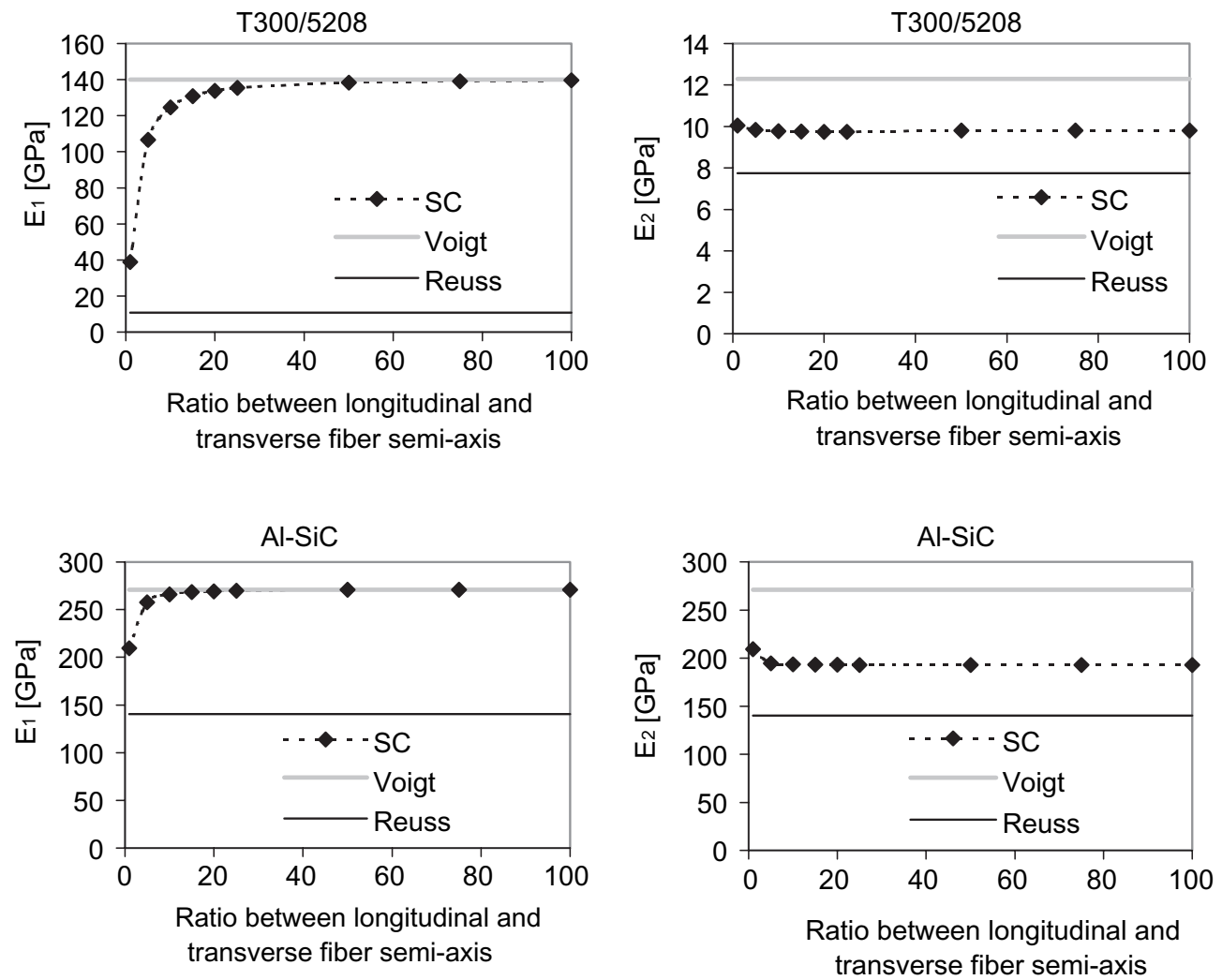

Figure 1. Evolutions of longitudinal and transverse elastic moduli estimated at the macroscopic scale in various composite materials. Dependence with inclusion morphology. 
Table 1. Mechanical properties of the constituents of the studied composites.

\begin{tabular}{lccccc}
\hline & $E_{\mathbf{1}}(\mathrm{GPa})$ & $\boldsymbol{E}_{\mathbf{2}}, \boldsymbol{E}_{\mathbf{3}}(\mathrm{GPa})$ & $v_{\mathbf{1 2}}, \nu_{\mathbf{1 3}}$ & $\boldsymbol{G}_{\mathbf{2 3}}(\mathrm{GPa})$ & $\boldsymbol{G}_{\mathbf{1 2}}(\mathrm{GPa})$ \\
\hline T300 fibers [23] & 230 & 15 & 0.2 & 7 & 15 \\
N5208 epoxy matrix [24] & 4.5 & 4.5 & 0.4 & 1.6 & 1.6 \\
SiC & 402.1 & 402.1 & 0.182 & 170.1 & 170.1 \\
Aluminum & 71.0 & 71.0 & 0.346 & 26.37 & 26.37 \\
\hline
\end{tabular}

Table 2. Mechanical properties at the macroscopic scale deduced from SC computations, in the three studied composites, for the reference morphology $\left(a_{1}=100, a_{2}=1, a_{3}=1\right)$.

\begin{tabular}{lccccc}
\hline & $E_{1}(\mathrm{GPa})$ & $E_{2}, E_{3}(\mathrm{GPa})$ & $\nu_{12}, \nu_{13}$ & $G_{23}(\mathrm{GPa})$ & $G_{12}(\mathrm{GPa})$ \\
\hline T300/5208 & 139.6 & 9.8 & 0.28 & 3.5 & 6.4 \\
Al-SiC & 271 & 192.8 & 0.23 & 74.1 & 83.1 \\
\hline
\end{tabular}

material. Calculations were performed assuming the initial local elastic properties listed in Table 1, and a fiber volume fraction of $60 \%$ (the "pseudo-macroscopic" elastic behavior taken into account for aluminum and $\mathrm{SiC}$ was deduced from a classical single-phase isotropic SC scale transition model [4], involving the single-crystal properties of each phase given by Chung and Buessem in [25]).

The results obtained with SC model for the elastic properties of the composites remain between the classical upper and lower bounds respectively given by Voigt and Reuss assumptions. Transverse elastic moduli of composites estimated according to the SC formalism do not noticeably vary with the length of the longitudinal semi-axis chosen for the inclusions. On the contrary, the longitudinal macroscopic modulus deduced according to the SC estimates strongly depends on the morphology considered for the inclusions. Voigt estimation is reached further in the case of the carbon-epoxy composite than in the MMC (i.e. for a ratio between longitudinal and transverse inclusion semi-axis of 100 , compared to the values of 50 obtained for the MMC). It is usually considered that the longitudinal elastic modulus of a ply should be close to the one predicted by the Voigt model, for a resin reinforced by fibers. In consequence, the morphologic ratio of 100 is kept for the needs of CME simulations and furthermore, local stresses and strains calculations. Table 2 summarizes the overall elastic properties of the ply for this particular morphology.

\section{EVALUATION OF THE OVERALL CME OF A PLY IN A T300/5208 COMPOSITE USING SELF-CONSISTENT MODEL}

The predictive integral forms (14) and (15) demonstrated for the homogeneous equivalent $\mathrm{CME}$ of a ply, according to the self-consistent scale transition formalism developed in the present work, was checked in the case of a T300/5208 lamina. CME for the constituents are given in Table 3. Calculations have been performed for several moisture content ratios $\left(\Delta C^{m}\right) /\left(\Delta C^{I}\right)$ varying from 1 to the maximum moisture content ratio.

In the case studied, the ratio between the composite and the resin densities being 1.33, the maximum moisture content ratio given by (17) is about 3.33. Ply CME estimated through the SC model are compared to the corresponding values predicted by Tsai-Hahn forms (30) and (31). Results obtained for the longitudinal and transverse macroscopic 
Table 3. CME for the components of T300/5208 composite.

\begin{tabular}{lll}
\hline & $\beta_{\mathbf{1 1}}$ & $\beta_{\mathbf{2 2}}, \beta_{\mathbf{3 3}}$ \\
\hline T300 fibers [23] & 0 & 0 \\
N5208 epoxy matrix [24] & 0.6 & 0.6 \\
\hline
\end{tabular}
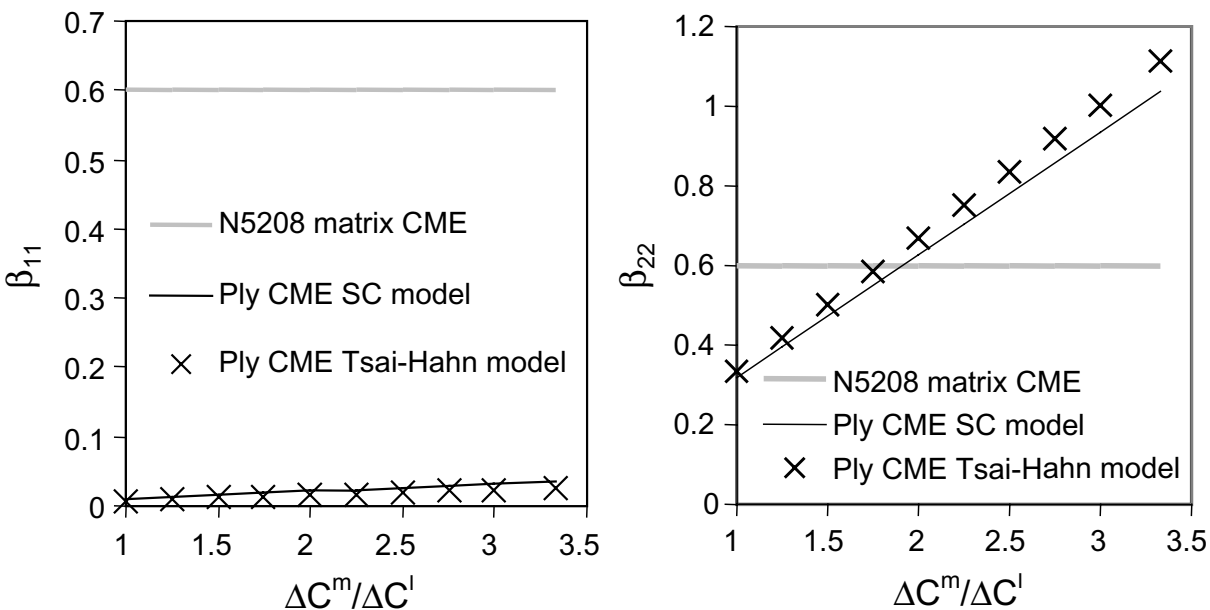

Figure 2. Longitudinal and transverse CME estimated for T300/5208 composite. Comparison between SC and Tsai-Hahn models.

CME have been shown in Figure 2. The SC model yields satisfactory numerical values in comparison with Tsai-Hahn reference CME. This demonstrates the reliability of SC approach to simulate the equivalent hygroelastic properties of the composite materials. Computations show that SC formalism succeeds in reproducing a strongly anisotropic hygroscopic behavior at the macroscopic scale: the longitudinal CME of the ply remains many times weaker than that of the epoxy matrix, whatever the assumed moisture content ratio. On the contrary, transverse macroscopic CME can be either higher or weaker than resin CME. As predicted by Tsai-Hahn forms, the CME calculated owing to the SC model can be stronger in the ply than in the epoxy matrix, especially for high values of $\left(\Delta C^{m}\right) /$ $\left(\Delta C^{I}\right)$. This corresponds to quasi-perfect materials, i.e. nonporous matrix and no adhesion defects at its interface with the fiber. Transverse macroscopic CME significantly increases with moisture content ratio. In the studied case, T300/5208 CME reaches the values observed in the resin for $\left(\Delta C^{m}\right) /\left(\Delta C^{I}\right) \approx 2$.

Self-consistent new predictive relations for the simulation of $\mathrm{CME}$ in composite materials give satisfactory results. In the following section, the SC model proposed in the present paper will be applied to the determination of the local mechanical states, either in the matrix or in the fibers of T300/5208 materials.

\section{Local and Macroscopic Stress States}

\section{MACROSCOPIC STRESS STATE}

A thin unidirectionally reinforced composite pipe, with $4 \mathrm{~mm}$ thickness, initially dry then exposed to an ambient fluid, made up of T300/5208 carbon-epoxy plies is considered. 
The use of the Tsai strength criterion allows to estimate the macroscopic internal stresses versus the ply resistance. The macroscopic tensile failure stresses in the longitudinal, normal, and transverse directions denoted respectively $X, Y$, and $Z$ are reported in Table 4. The corresponding macroscopic failure stresses for compression are $X^{\prime}, Y^{\prime}, Z^{\prime}$, and $S$ stands for the macroscopic plane shear failure stress. These strengths are assumed not to depend on humidity. $R$, the strength factor derived from the Tsai strength criterion, stands for the ratio between the ultimate stress and the applied stress. Therefore, the failure occurs when $R$ is less than or equal to 1 .

Tables 1 and 3 present typical data for the properties of the T300 carbon fibers and N5208 epoxy matrix. The mechanical properties of the T300/5208 composite, deduced from the SC computations, are presented in Table 2. The hygroscopic properties are presented in Table 5 .

Figure 3 shows the time-dependent concentration profiles, resulting from the application of a boundary concentration $c_{0}$, as a function of the normalized radial distance from the inner radius $r_{\mathrm{dim}}$. At the beginning of the diffusion process, important concentration gradients occur near the external surfaces. The permanent concentration (noticed

Table 4. Strengths.

\begin{tabular}{lccccr}
\hline Strengths (MPa) & $X$ & $X^{\prime}$ & Y, Z & $\mathbf{Y}^{\prime}, \mathbf{Z}^{\prime}$ & $\mathbf{S}$ \\
\hline T300/5208 [24] & 1500 & 1500 & 40 & 246 & 68 \\
\hline
\end{tabular}

Table 5. Hygroscopic properties for T300/5208 composite [13].

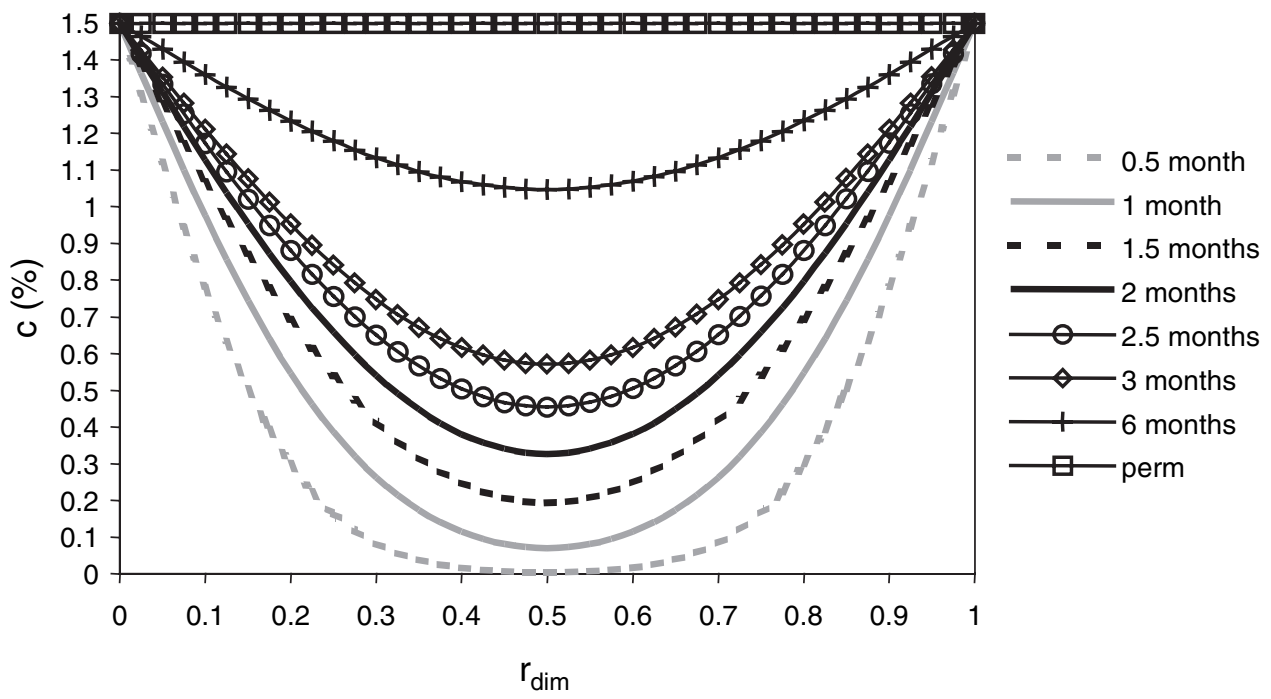

Figure 3. T300/5208 concentration profiles. 
'perm' in the caption) holds with a constant value because of the symmetric hygroscopic loading.

Figure 4 shows the transverse macroscopic stresses for moisture content ratios of 1 , $1.5,2$, and 3.33, respectively. As time advances, the transverse stress in the central ply increases, attaining its maximum value after a short time-interval, then decreases gradually as the concentration distribution (Figure 3) approaches its uniform equilibrium level. The maximum transverse stress, reached in the central ply, is a growing function of the moisture content ratio. The transverse stresses for a moisture content ratio of 2 (Figure 4(c)) reach positive values which are about $50 \%$ of the transverse tensile strength (Table 4). Critical values of the strength factor (Figure 5(b)), less than 1, are reached for the maximum moisture content ratio. Actually, for this moisture content ratio, the transverse stress exceeds the macroscopic tensile strength in the transverse direction (Figure 4(d)).
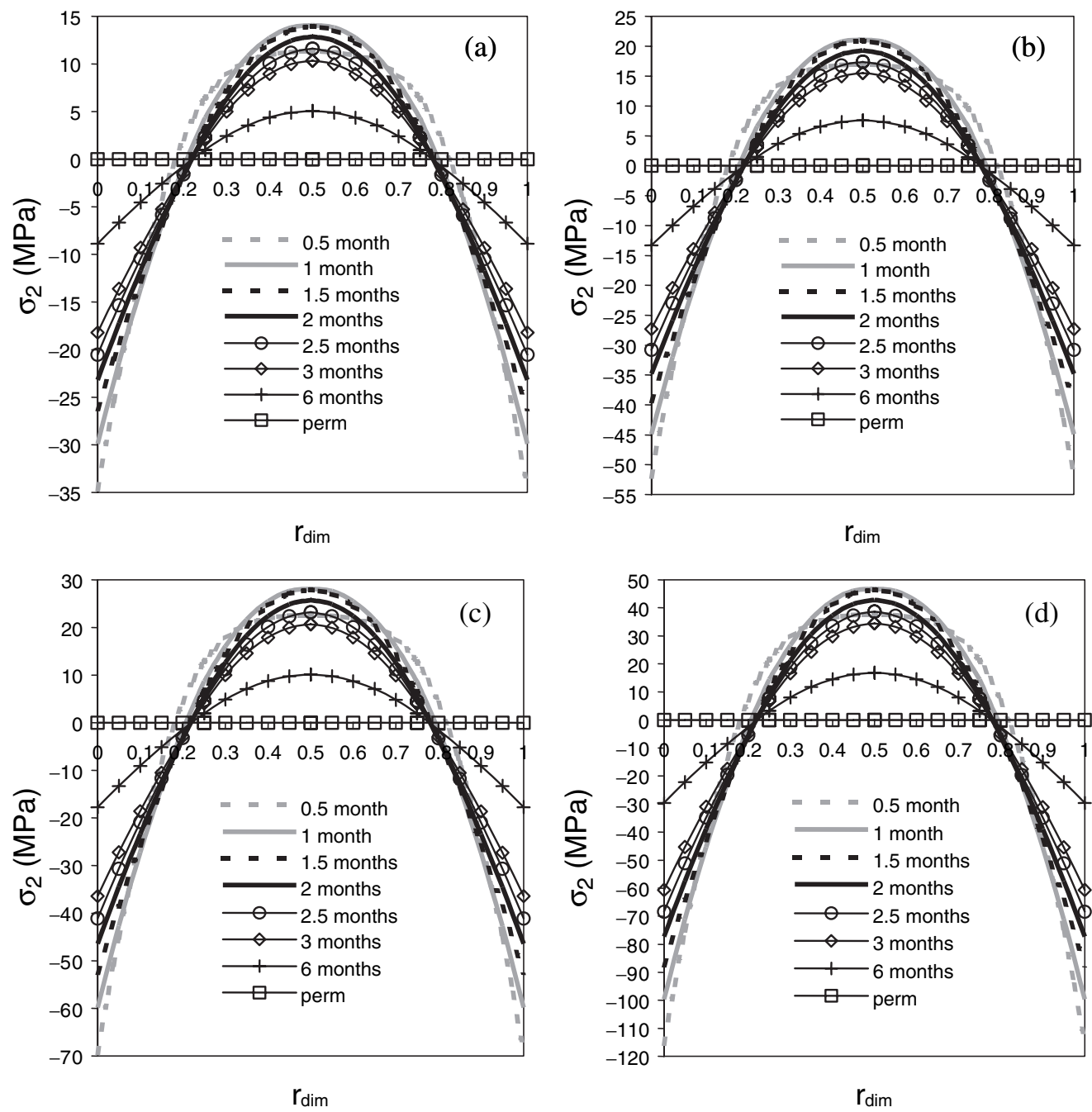

Figure 4. T300/5208 transverse macroscopic stresses for moisture content ratio of (a) 1; (b) 1.5; (c) 2; and (d) 3.33 . 
(a)

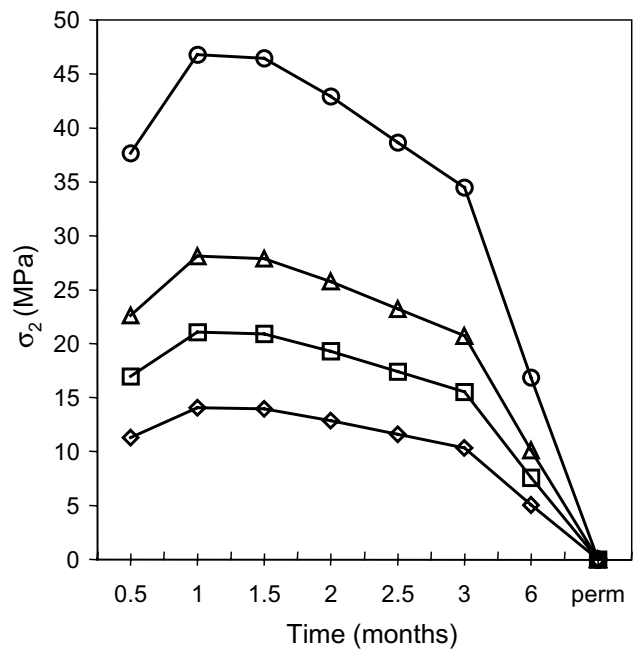

(b)

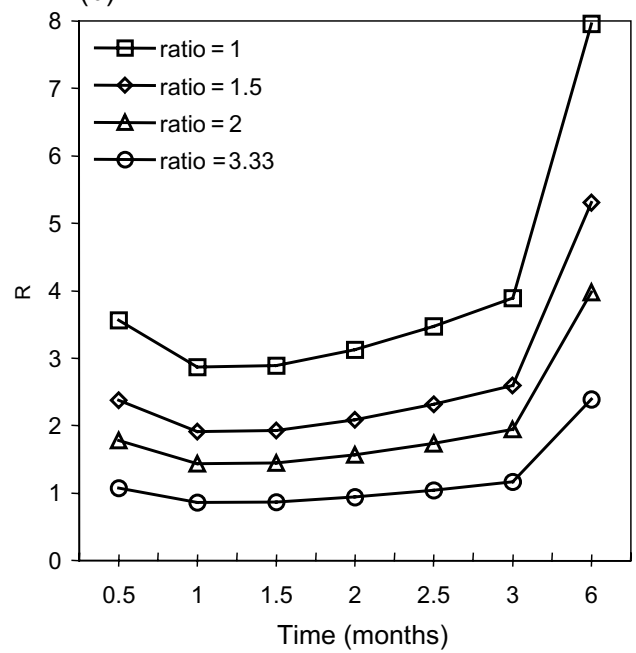

Figure 5. (a) Transverse stresses in T300/5208 central ply and (b) strength factor in T300/5208 central ply.

(a)

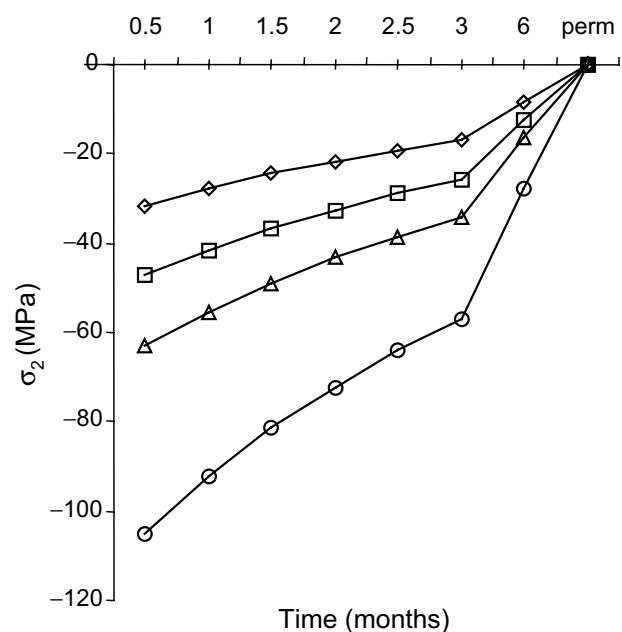

(b)

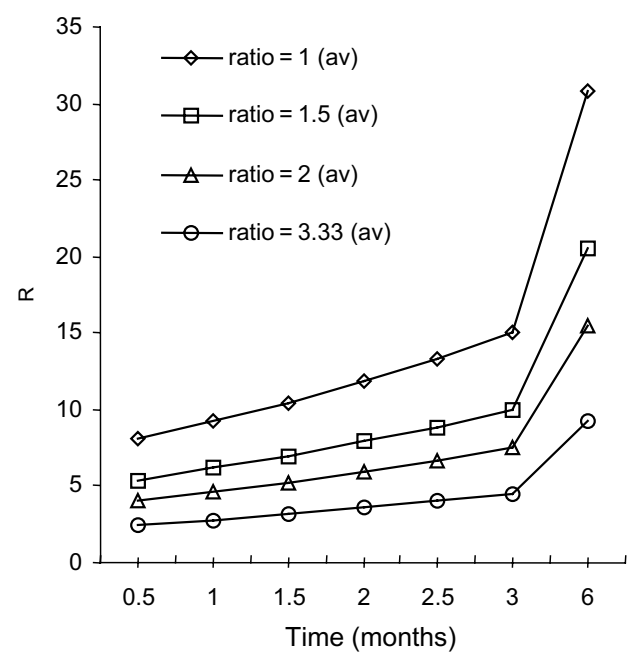

Figure 6. (a) Average transverse macroscopic stresses in T300/5208 inner ply and (b) strength factor in T300/ 5208 inner ply.

The transient analysis emphasizes the existence of minimum strength factors in the first times.

In the inner ply, the macroscopic transverse stress is not constant. The average transverse macroscopic stresses and the corresponding strength factors in the inner ply are plotted in Figure 6(a) and (b). The transverse stresses (Figure 6(a)) remain negative whatever the moisture content ratio. For the maximum moisture content ratio, the transverse stresses do not reach the compressive strength in the transverse direction. Thus no failure occurs and the strength factor remains higher than 1 (Figure 6(b)). Finally, macroscopically a tensile failure occurs in the central ply. 


\section{LOCAL STRESS STATES}

By assuming that the fibers do not absorb any moisture, the local stresses-strains relation in reinforcements (2) becomes:

$$
\boldsymbol{\sigma}^{f}=\mathbf{L}^{f}: \boldsymbol{\varepsilon}^{f}
$$

Using (39) in (1) and (3) we obtain the following scale transition expression for the strains in fibers:

$$
\boldsymbol{\varepsilon}^{f}=\left(\mathbf{L}^{f}+\mathbf{L}^{I}: \mathbf{R}^{I}\right)^{-1}:\left(\boldsymbol{\sigma}^{I}+\mathbf{L}^{I}: \mathbf{R}^{I}: \boldsymbol{\varepsilon}^{I}\right)
$$

The use of (40) implies the knowledge of the macroscopic stresses and strains in the ply. This especially requires the measurement or calculation of the CME of the composite material.

If these conditions are satisfied, the local mechanical states in the epoxy matrix are provided by Hill's strains and stresses average laws (5):

$$
\begin{gathered}
\boldsymbol{\sigma}^{m}=\frac{1}{v^{m}} \boldsymbol{\sigma}^{I}-\frac{v^{f}}{v^{m}} \boldsymbol{\sigma}^{f} \\
\boldsymbol{\varepsilon}^{m}=\frac{1}{v^{m}} \boldsymbol{\varepsilon}^{I}-\frac{v^{f}}{v^{m}} \boldsymbol{\varepsilon}^{f}
\end{gathered}
$$

Forms (39)-(42) depend on neither the local CME of the matrix nor its moisture content.

The transverse stresses in the matrix and fiber, corresponding to the macroscopic stresses in the central ply, for the different moisture content ratios are plotted in Figure 7 (a) and (b), respectively. The transverse stresses in the matrix and fiber are growing functions of the moisture content ratio. For the maximum moisture content ratio, the
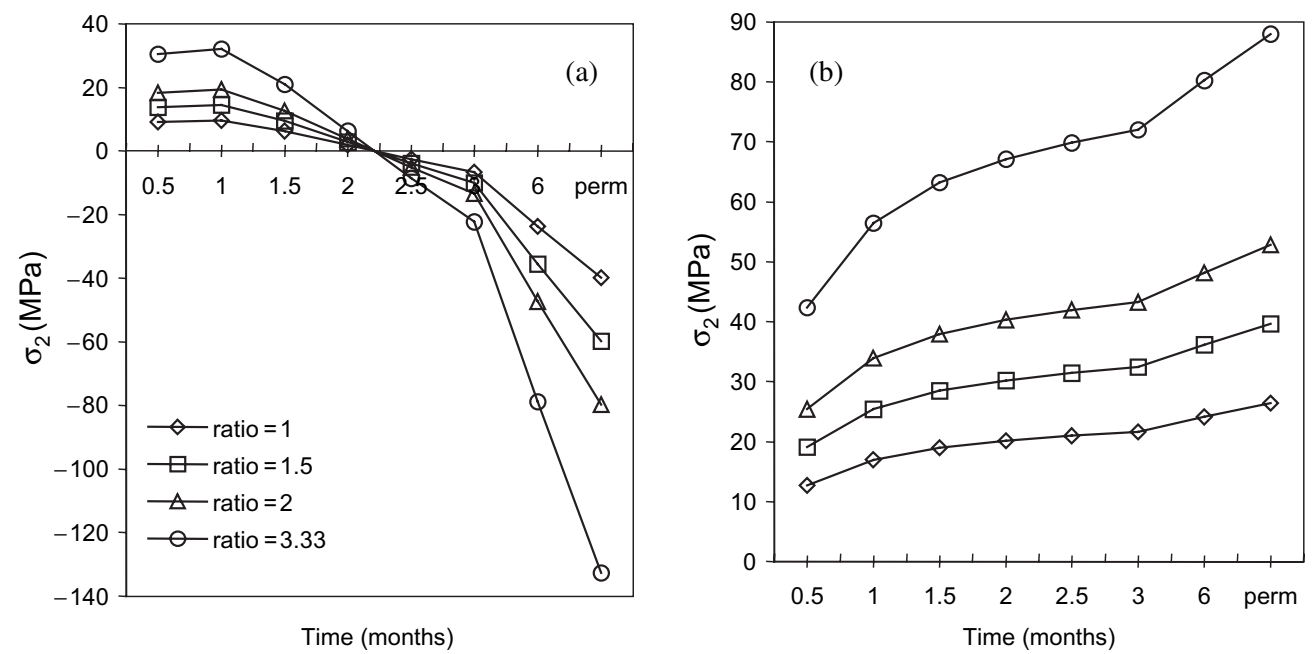

Figure 7. (a) Transverse stresses in N5208 matrix (central ply) and (b) transverse stresses in T300 fiber (central ply). 

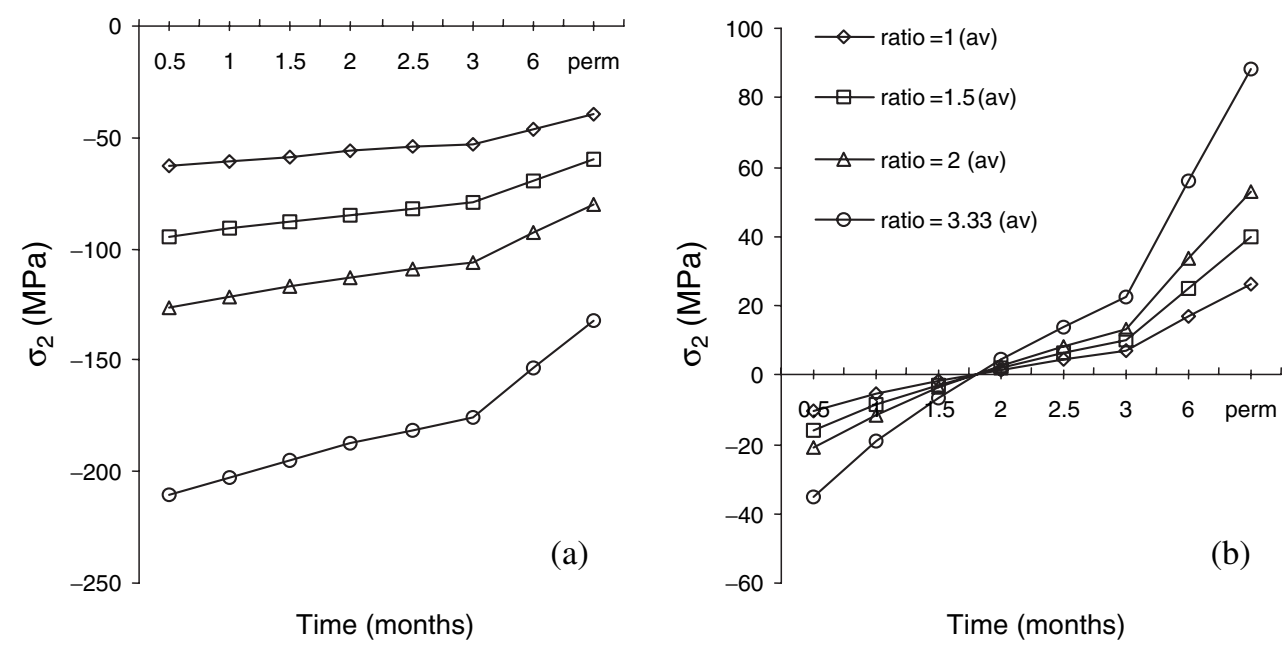

Figure 8. (a) Transverse stresses in N5208 matrix (inner ply) and (b) transverse stresses in T300 fiber (inner ply).

transverse stress in the matrix (Figure 7(a)) varies with time from tensile values superior to $30 \mathrm{MPa}$ (first stages) to compressive values around $130 \mathrm{MPa}$ (corresponding to the permanent state).

The transverse stresses in the matrix and fiber, corresponding to the average macroscopic stress on the inner ply, for the different moisture content ratios are plotted in Figure 8(a) and (b), respectively. The transverse stress in the matrix remains negative whatever the moisture content ratio. The maximum transverse compressive stress is reached at the first time and for a ratio of 3.33; the stress values could reach the compressive strength of the matrix while macroscopically a transverse tensile failure occurs in the central ply. The stress in the fiber is initially compressive and as time advances it is tensile.

\section{CONCLUSIONS}

A hygroelastic self-consistent model for unidirectional fiber-reinforced composites subjected to transient moisture concentration has been investigated in this paper. The moisture contents in the matrix and fiber are strongly different, accordingly the classical Self-consistent approach is extended in order to predict the macroscopic elastic properties and the Coefficients of Moisture Expansion (CME). Inverse forms are proposed to determine the unknown CME of a given matrix. Elastic constants and CME predicted have been compared to the Reuss-Voigt estimates and the Tsai-Hahn model, respectively. An imperfect adhesion in fiber-reinforced composites and a porous matrix induce various ratios between the moisture content of the neat resin and the moisture content of the composite. The macroscopic (ply) and local (fiber and matrix) internal stress states are evaluated for various moisture content ratios between the matrix and the ply. The macroscopic stresses are calculated by using the continuum mechanics formalisms and the local stresses derived from the scale transition model. Finally, a macroscopic strength criterion is used to estimate the internal stress state versus ply resistance for different hygroscopic conditions. It is shown, for maximum moisture content ratio, corresponding to a perfect adhesion between the fiber and a nonporous matrix, that a macroscopic tensile 
failure occurs in the central ply of the composite. On the contrary, scale transition model enlightens a possible damage due to compressive stresses in the matrix for the inner ply. This demonstrates the complementarities between the continuum mechanics and scale transition modeling for the study of heterogeneous materials such as composites.

\section{REFERENCES}

1. Kröner, E. (1958). Berechnung der elastischen Konstanten des Vielkristalls aus des Konstanten des Einkristalls, Zeitschrift für Physik, 151: 504-518.

2. François, M. (1991). Détermination de contraintes résiduelles sur des fils d'acier eutectoïde de faible diamètre par diffraction des rayons X, Doctoral Thesis, ENSAM, Paris.

3. Mabelly, P. (1996). Contribution à l'étude des pics de diffraction - Approche expérimentale et modélisation micromécanique, Doctoral Thesis, ENSAM, Aix en Provence.

4. Kocks, U. F., Tomé, C. N. and Wenk, H. R. (1998). Texture and Anisotropy, Cambridge University Press, Cambridge, UK.

5. Hutchinson, J. W. (1970). Elastic-Plastic Behaviour of Polycrystalline Metals and Composites, In: Proceedings of the Royal Society London, 319, pp. 247-272.

6. Turner, P. A. and Tome, C. N. (1994). A Study of Residual Stresses in Zircaloy-2 with Rod Texture, Acta Metallurgica and Materialia, 42: 4143-4153.

7. Gloaguen, D., François, M., Guillén, R. and Royer, J. (2002). Evolution of Internal Stresses in Rolled $\mathrm{Zr702} \alpha$, Acta Materialia, 50: 871-880.

8. Fréour, S., Gloaguen, D., François, M. and Guillén, R. (2003). Study of the Coefficients of Thermal Expansion of Phases Embedded in Multiphase Materials, Materials Science Forum, 426-432: 2083-2088.

9. Sprauel, J. M. and Castex, L. (1991). Proceedings of the First European Powder Diffraction International Conference on X-Ray Stress Analysis, Munich.

10. Eshelby, J. D. (1957). The Determination of the Elastic Field of an Ellipsoidal Inclusion, and Related Problems, In: Proceedings of the Royal Society London, A241, pp. 376-396.

11. Hill, R. (1965). Continuum Micro-mechanics of Elastoplastic Polycrystals, Journal of the Mechanics and Physics of Solids, 13: 89-101.

12. Hill, R. (1967). The Essential Structure of Constitutive Laws for Metals Composites and Polycrystals, Journal of the Mechanics and Physics of Solids, 15: 79-95.

13. Loos, A. C. and Springer, G. S. (1981). Environmental Effects on Composite Materials, Moisture Absorption of Graphite - Epoxy Composition Immersed in Liquids and in Humid Air, pp. 34-55, Technomic Publishing, Westport, USA.

14. Anifantis, N. K., Kakavas, P. A. and Papanicolaou, G. C. (1997). Thermal Stress Concentration due to Imperfect Adhesion in Fiber-reinforced Composites, Composite Science and Technology, 57: 687-696.

15. Mensitieri, G., Del Nobile, M. A., Apicella, A. and Nicolais, L. (1995). Moisture-Matrix Interactions in Polymer Based Composite Materials, Revue de l'Institut Francais du Pétrole, 50: 551-571.

16. Voigt, W. (1928). Lehrbuch der Kristallphysik, Teubner, Leipzig/Berlin.

17. Reuss, A. (1929). Berechnung der Fliessgrenze von Mischkristallen auf Grund der Plastizitätsbedingung für Einkristalle, Zeitschrift für Angewandte und Mathematik und Mechanik, 9: 49-58.

18. Chamis, C. C. (1984). Simplified Composite Micromechanics Equations for Strength, Fracture Toughness, and Environmental Effects, NASA Report TM-83696.

19. Gibson, R. F. (1994). Principles of Composite Material Mechanics, McGraw Hill, New York.

20. Tsai, S. W. and Hahn, H. T. (1980). Introduction to Composite Materials, Technomic Publishing Co., Inc., Lancaster, Pennsylvania.

21. Crank, J. (1975). The Mathematics of Diffusion, Clarendon Press, Oxford.

22. Jacquemin, F. and Vautrin, A. (2002). A Closed-form Solution for the Internal Stresses in Thick Composite Cylinders Induced by Cyclical Environmental Conditions, Composite Structures, 58: 1-9.

23. Soden, P. D., Hinton, M. J. and Kaddour, A. S. (1998). Lamina Properties Lay-up Configurations and Loading Conditions for a Range of Fiber-reinforced Composite Laminates, Composites Science and Technology, 58: 1011-1022.

24. Tsai, S. W. (1987). Composite Design, 3rd edn, Think Composites, Dayton, USA.

25. Chung, D. H. and Buessem, W. R. (1967). The Elastic Anisotropy of Crystals, Journal of Applied Physics, 39: 217-245. 